\title{
WHERE DO WE STAND ON CAPGRAS: FROM SEPARATED DELUSIONS TO NEUROPSYCHIATRY
}

\section{Felizardo ${ }^{1}$, I. Vaz¹, D. Maia1}

${ }^{1}$ Centro-Hospitalar Tras-os-Montes-e-alto-Douro, Psychiatry, Vila Real, Portugal.

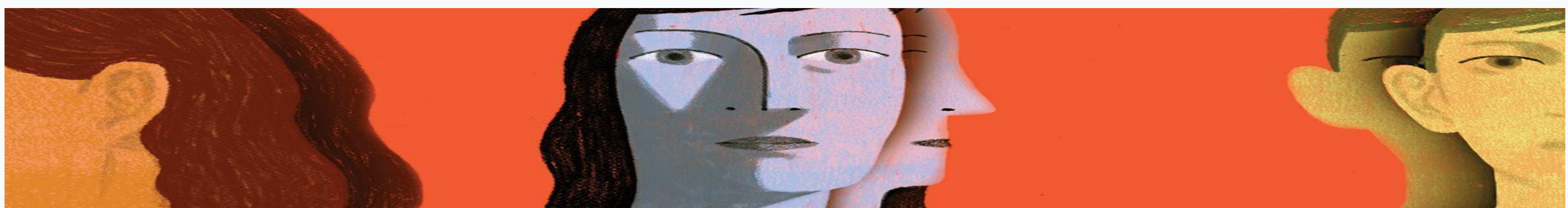

\section{Background:}

Capgras syndrome is the most prevalent of the delusional misidentification syndromes. It is characterized by the delusional belief that a family member, friend, or loved one has been replaced by an imposter. Capgras' delusion has captured psychiatrists' imaginations, but the clinical features of the delusion have rarely been studied.

\section{Objectives:}

Our main goals are to portrait Capgras syndrome etiology and to construct a review of the current psychopathological and neurobiological theories based on clinical and neuroanatomy findings throughout the years.

\section{Discussion:}
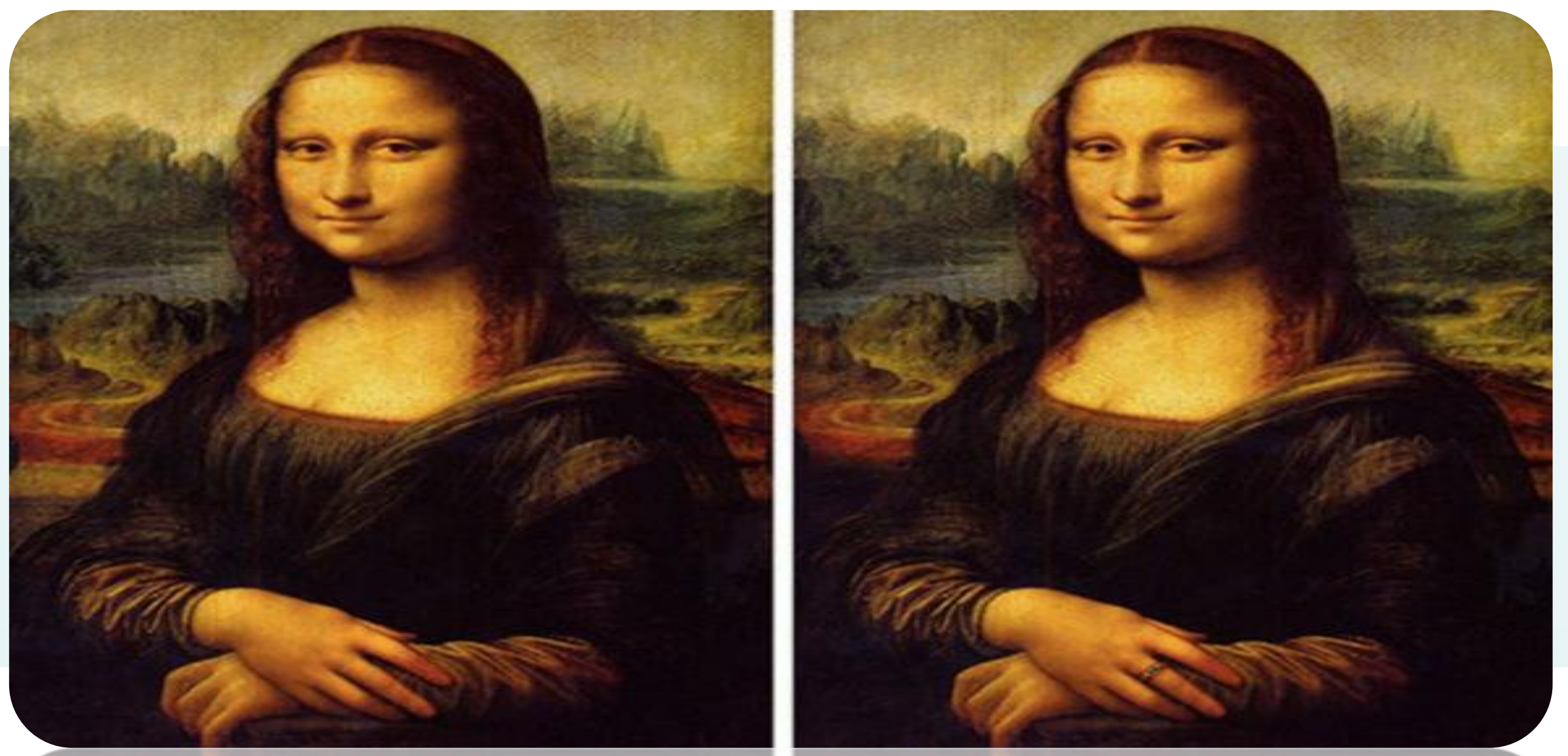

Joseph Capgras used the term l'illusion dês sosies to describe the case of a woman who complained of several women took the place of people she knew. The subsequent cognitive neuropsychiatric explanation has received considerable attention from psychiatrists, neuropsychologists, and philosophers alike; it oftentimes appears to be regarded the Rosetta stone to open the way to an explanation of all delusions. Indeed, Daniel Dennett, a renowned philosopher of neuroscience described Capgras' delusion as an "amazing phenomenon (that) should send shock waves through philosophy. In psychodynamic terms, early attempts to explain Capgras syndrome invoked the Edipus and Electra complex or inappropriately repressed thoughts to whom the delusional content refers. According to cognitive models, Capgras syndrome cannot be exclusively conceived as a dysfunction in facial recognition but in recognizing a person globally considered. From the neuropsychiatry point of view, Capgras delusion arises from the disconnection between frontal lobes and right temporo-limbic regions.

\section{Conclusions:}

When we talk about Capgras syndrome, we must include clinical, neuropshychiatric and neuropsychological approaches. Many theories have attempted to explain how the abnormal belief in Capgras syndrome emerges. It is still early to assume we have a solid work to define how Capgras specific delusions come into place and why, but neuropsychological hypotheses based on cerebral dysfunctions are now commonly considered to be at the origin of the disorder. 